\title{
Races of Puccinia graminis f. sp. tritici with Combined Virulence to Sr13 and Sr9e in a Field Stem Rust Screening Nursery in Ethiopia
}

\author{
P. D. Olivera, Department of Plant Pathology, University of Minnesota, St. Paul, MN 55108; Y. Jin and M. Rouse, United States \\ Department of Agriculture-Agricultural Research Service, Cereal Disease Laboratory, St. Paul, MN 55108; A. Badebo, Ethiopian \\ Institute of Agricultural Research, Debre Zeit, Ethiopia; T. Fetch, Jr., Cereal Research Center, Agriculture and Agri-Food Canada, \\ Winnipeg, MB R3T 2M9, Canada; and R. P. Singh and A. Yahyaoui, International Maize and Wheat Improvement Center, El Batan, \\ Mexico, CP 56130
}

\begin{abstract}
Olivera, P. D., Jin, Y., Rouse, M., Badebo, A., Fetch, T., Jr., Singh, R. P., and Yahyaoui, A. 2012. Races of Puccinia graminis f. sp. tritici with combined virulence to $\operatorname{Sr} 13$ and $S r 9 e$ in a field stem rust screening nursery in Ethiopia. Plant Dis. 96:623-628.

North American durum lines, selected for resistance to TTKSK (Ug99) and related races of Puccinia graminis f. sp. tritici in Kenya, became susceptible in Debre Zeit, Ethiopia, suggesting the presence of stem rust races that were virulent to the TTKSK-effective genes in durum. The objective of this study was to characterize races of $P$. graminis $\mathrm{f}$. sp. tritici present in the Debre Zeit, Ethiopia stem rust nursery. Three races of $P$. graminis f. sp. tritici were identified from 34 isolates: JRCQC, TRTTF, and TTKSK. Both races JRCQC and TRTTF possess virulence on stem rust resistance genes $\operatorname{Sr} 13$ and $\operatorname{Sr} 9 e$, which may explain why many TTKSK-resistant durum lines tested in Kenya became susceptible in Debre Zeit. The $\mathrm{Sr} 9 e-\mathrm{Sr} 13$ virulence combination is of particular concern because these two genes constitute major components of stem rust resistance in North American durum cultivars. In addition to $\mathrm{Sr} 9 e$ and $\mathrm{Srl3}$ virulence, race TRTTF is virulent to at least three stem rust resistance genes that are effective to race TTKSK,

including $\operatorname{Sr} 36$, $\operatorname{SrTmp}$, and resistance conferred by the 1AL.1RS rye translocation. Race TRTTF is the first known race with virulence to the stem rust resistance carried by the 1AL.1RS translocation, which represents one of the few effective genes against TTKSK in winter wheat cultivars in the United States. Durum entries exhibiting resistant to moderately susceptible infection response at the Debre Zeit nursery in 2009 were evaluated for reaction to races JRCQC, TRTTF, and TTKSK at the seedling stage. In all, 47 entries were resistant to the three races evaluated at the seedling stage, whereas 26 entries exhibited a susceptible reaction. These results suggest the presence of both major and adult plant resistance genes, which would be useful in durumwheat-breeding programs. A thorough survey of virulence in the population of $P$. graminis f. sp. tritici in Ethiopia will allow characterization of the geographic distribution of the races identified in the Debre Zeit field nursery.
\end{abstract}

Stem rust, caused by Puccinia graminis f. sp. tritici, is one of the most destructive diseases of bread wheat (Triticum aestivum), durum wheat (T. turgidum subsp. durum), and barley (Hordeum vulgare). Races that recently emerged in eastern Africa (TTKSK [Ug99] and its variants) possess broad virulence to wheat cultivars worldwide, and only a few genes in adapted cultivars are effective against these races $(7,8,21)$. Durum wheat in North America generally has a higher frequency of resistance to race TTKSK than common wheat based on field evaluations conducted in Njoro, Kenya in 2005 and 2006 (R. Singh and Y. Jin, unpublished). Pozniak et al. (14) described that over $80 \%$ of the durum varieties and breeding lines from breeding programs in the United States and Canada evaluated in a field trial in Njoro exhibited a moderately resistant or resistant response to stem rust. Singh et al. (18) also reported that durum lines from Egypt and the International Maize and Wheat Improvement Center (CIMMYT) exhibited a high level of resistance to races TTKSK and TTKST in the Njoro's field nursery. However, when durum lines from the United States and CIMMYT were selected for resistance in Kenya and subsequently were evaluated in Debre Zeit, Ethiopia, many became susceptible to stem rust (R. Singh and Y. Jin, unpublished). Thus, we hypothesized that races of $P$. graminis $\mathrm{f}$. sp. tritici in the Debre Zeit nursery possess virulence that overcomes the TTKSK resistance in North

Corresponding author:Y. Jin, E-mail: yue.jin@ars.usda.gov

Accepted for publication 10 November 2011.

http://dx.doi.org/10.1094/PDIS-09-11-0793

This article is in the public domain and not copyrightable. It may be freely reprinted with customary crediting of the source. The American Phytopathological Society, 2012.
American durum germplasm. The objective of this study was to identify the race or races of $P$. graminis f. sp. tritici present in the Debre Zeit, Ethiopia nursery that are virulent on durum lines with TTKSK resistance.

\section{Materials and Methods}

Stem rust nursery. The 2009 durum stem rust field nursery was established by the Ethiopian Institute of Agricultural Research at the Debre Zeit Research Center in Ethiopia. The nursery site was located at $08^{\circ} 46^{\prime} \mathrm{N}, 39^{\circ} 00^{\prime} \mathrm{E}$, and $1,900 \mathrm{~m}$ in elevation. Entries were planted in double 1-m-row plots on 15 July 2009. Wheat 'Red Bobs' (CItr 6255) was included at an interval of 50 lines as a susceptible control. Continuous rows of stem rust spreader (mixture of susceptible cultivars) were planted perpendicular to all entries to facilitate inoculum build-up and uniform dissemination. The spreader rows were planted the same day as the wheat entries and were artificially inoculated by needle injection three times at a weekly interval, starting at stem elongation (stage $=$ Zadoks 31 ) (23). Urediniospores were suspended in distilled water plus one drop of Tween 20 per 0.5 liters of suspension, and delivered with a hypodermic syringe into the stem tissue. Inoculum was composed of race TTKSK and a bulk of Ethiopian isolates (with unknown race identities) at a ratio of 50/50. Race TTKSK was originally collected from wheat 'PBW343' (carrying Sr31) at Debre Zeit, and the bulk of Ethiopian isolates was collected from durum lines at the Debre Zeit Research Center.

Sample collection and storage. Forty-one samples of infected stems were collected from durum and common wheat lines with known $\mathrm{Sr}$ genes in the stem rust field nursery in 2009. Each sample consisted of 10 to 15 pieces of stem tissue of about $10 \mathrm{~cm}$ in length bearing moderately susceptible to susceptible pustules. Stem and leaf sheath tissue were kept in glassine bags and air dried for 2 days at room temperature in the dark. Dried samples were mailed 
to the United States using an international express courier service with a transit time of 5 days. Shipping protocol was followed according to United States Department of Agriculture (USDA) Animal and Plant Health Inspection Service permit conditions for handling international cultures of $P$. graminis f. sp. tritici. Upon arrival at the Cereal Disease Laboratory, samples were stored in a $80^{\circ} \mathrm{C}$ freezer. In December, urediniospores from each stem sample were collected into three to four gelatin capsules (size 00) and stored at $-80^{\circ} \mathrm{C}$.

Race identification. The North American stem rust differential set $(15,16)$ that was modified to further delineate races in the TTKS race group (9) was used for race identification. One capsule per sample was removed from the $-80^{\circ} \mathrm{C}$ freezer and exposed to a heat-shock treatment (water bath at $45^{\circ} \mathrm{C}$ for $15 \mathrm{~min}$ ), then placed in a rehydration chamber $(80 \%$ relative humidity maintained by a $\mathrm{KOH}$ solution) for a period of $4 \mathrm{~h}$ (9). Five seedlings from each of the 20 differential lines were inoculated with a bulk collection of spores on fully expanded primary leaves at 8 to 9 days after planting. Experimental procedures for inoculation, incubation, and disease assessment were done as described by Jin et al. (8). Singlepustule isolates were derived from individual plants after preliminary evaluation on the differential lines. Six to eight pustules were isolated from each original collection. Incubation and collection of urediniospores from each single pustule was done as described by Jin et al. (9). The pure cultures derived from single-pustule inoculation were increased on 'McNair 701' wheat (CItr 15288) and stored at $-80^{\circ} \mathrm{C}$. Each single-pustule isolate was evaluated two to three times on differential lines before a race was designated. Race designation was done based on the letter code proposed by Roelfs and Martens (16). Representative isolates from each race were further characterized on 17 additional monogenic lines carrying the following genes: $\operatorname{Sr} 22, \operatorname{Sr} 25, \operatorname{Sr} 26, \operatorname{Sr} 27, \operatorname{Sr} 32, \operatorname{Sr} 33, \operatorname{Sr} 35, \operatorname{Sr} 37$, Sr39, Sr40, Sr42, Sr44, Sr46, Sr47, Sr50, SrSatu, and the 1A.1R rye translocation. Cultivars and lines carrying $\mathrm{Sr} 13$ and $\mathrm{Sr} 9 e$ alone or in combination and both resistant (Iumillo [PI 210973]) and susceptible (Rusty [PI 639869]) checks were also included in the evaluation of each isolate.

Seedling evaluation of wheat germplasm. One thousand durum entries deposited at the USDA Agricultural Research Service, National Small Grain Collection (Aberdeen, ID) were evaluated for resistance in field test at the 2009 durum stem rust field nursery in Debre Zeit. Disease assessment was done at the soft-dough stage of plant growth. Plants were evaluated for their infection response (pustule type and size) (17) and stem rust severity following the modified Cobb scale (13). In all, 137 durum wheat lines character- ized as resistant with a maximum $30 \%$ stem rust severity and maximum moderately susceptible infection response were evaluated at the seedling stage with $P$. graminis f. sp. tritici races identified from this nursery following procedures described above. Seedling evaluation was repeated once.

\section{Results and Discussion}

We obtained 34 single-pustule isolates from the 41 samples collected in 2009 from the Debre Zeit nursery. Three races of $P$. graminis f. sp. tritici were identified: JRCQC, TRTTF, and TTKSK (Table 1). The race most frequently observed was TTKSK (18 isolates), followed by JRCQC (12 isolates) and TRTTF (4 isolates). The high frequency of race TTKSK was likely due to artificial inoculation of $P$. graminis f. sp. tritici collected from PBW 343 in the 2009 Debre Zeit field nursery. The virulence profile of race TTKSK from Debre Zeit was identical to that of race TTKSK isolates found in Kenya $(9,10)$.

Race JRCQC produced high infection types (ITs) on differential lines carrying $\operatorname{Sr} 6, \operatorname{Sr} 9 a, \operatorname{Sr} 9 d, \operatorname{Sr} 9 e, \operatorname{Sr} 9 g, \operatorname{Sr} 11$, $S r 13 / 17, \operatorname{Sr} 21$, and $\operatorname{SrMcN}$ (Table 1). Race JRCQC produced a high IT on differential line Combination VII, which carries $\operatorname{Srl3}$ in addition to $\mathrm{Sr} 17$ and was virulent on other Srl3-lines (Table 2). Thus, this race possesses relatively rare virulence to both $S r 9 e$ and $S r 13$, a combination believed to be the main component of stem rust resistance in contemporary North American durum cultivars (11). Race JRCQC produced an IT = X- on W2691Sr10 (tester for Sr10). W2691Sr10 generally produces IT $=0$; to $; 1$ when inoculated with avirulent North American stem rust isolates, and IT $=\mathrm{X}$ is infrequent except for a few isolates from the Pacific Northwest (Y. Jin, unpublished). It is not known, however, whether the "X" type was due to $\mathrm{Sr} 10$ or a different gene in the W2691Sr10 background. Race JRCQC was avirulent to additional stem rust resistance genes tested in this study, except presumably $\mathrm{Sr} 42$ in Norin 40 (Table 2).

Race TRTTF was first identified from a stem rust collection from Yemen in 2006 (Y. Jin and A. Yahyaoui, unpublished), and was further isolated from stem rust collections in Ethiopia and Yemen in subsequent years (T. Fetch, unpublished). Race TRTTF is virulent on most of the stem rust resistance genes in the differential set, including $\mathrm{Sr} 9 e$ (Table 1). Virulence on $\mathrm{Sr} 13$ was determined based on high IT (IT 3+) on Combination VII, which carries $\mathrm{Sr} 13$ in addition to $\mathrm{Sr} 17$ (Table 1). Virulence on $\mathrm{Sr} 9 e$ and $\mathrm{Sr} 13$ was also confirmed in monogenic lines Vernal $(\mathrm{Sr} 9 \mathrm{e})$ and Khapstein/9*LMPG (Sr 13), respectively (Table 2). However, the low ITs observed on $\mathrm{K} 253 / 3 *$ Steinwedel/8*LMPG $(\operatorname{Sr} 9 e)$ and Leeds $(\operatorname{Sr} 9 e$ + Sr13) (Table 2) indicate that these lines may carry an additional

Table 1. Infection types (ITs) observed on stem rust differentials using races JRCQC, TRTTF, and TTKSK of Puccinia graminis f. sp. tritici collected from the 2009 Debre Zeit (Ethiopia) field nursery ${ }^{\mathrm{a}}$

\begin{tabular}{|c|c|c|c|c|}
\hline Gene & Line & JRCQC (09ETH08-3) & TRTTF (09ETH06-1) & TTKSK (09ETH05-3) \\
\hline $\operatorname{Sr} 5$ & ISr5-Ra & $; 1$ & 4 & 4 \\
\hline $\operatorname{Sr} 21$ & $\mathrm{CnS} \mathrm{T}$ mono deriv & 4 & 4 & $3+$ \\
\hline Sr9e & Vernstein & 4 & $3+$ & 4 \\
\hline$S r 7 b$ & ISr7b-Ra & 2 & $3+$ & 4 \\
\hline Srl1 & ISr11-Ra & 4 & 4 & $3+$ \\
\hline $\operatorname{Sr} 6$ & ISr6-Ra & $3+$ & $3+$ & 4 \\
\hline $\mathrm{Sr} 8 \mathrm{a}$ & ISr8a-Ra & $22+$ & 2 & 4 \\
\hline $\operatorname{Sr} 9 g$ & $\mathrm{CnSr} 9 \mathrm{~g}$ & 4 & 4 & 4 \\
\hline Sr36 & W2691SrTt-1 & 0 & $3+$ & 0 \\
\hline $\operatorname{Sr} 9 b$ & $\mathrm{~W} 2691 \mathrm{Sr} 9 \mathrm{~b}$ & $2+$ & 4 & 4 \\
\hline Sr30 & BtSr30Wst & $22-$ & 4 & 4 \\
\hline $\operatorname{Sr} 17(+\operatorname{Sr} 13)$ & Combination VII & 3 & $3+$ & $22+$ \\
\hline $\operatorname{Sr} 9 a$ & ISr9a-Ra & $3+$ & 4 & $3+$ \\
\hline Sr9d & ISr9d-Ra & 4 & 4 & 4 \\
\hline $\operatorname{Sr} 10$ & W2691Sr10 & $1+3$ & 4 & 4 \\
\hline SrTmp & CnsSrTmp & $2-$ & 4 & $2+$ \\
\hline $\operatorname{Sr} 24$ & $\mathrm{LcSr} 24 \mathrm{Ag}$ & $2-$ & 2 & $2-$ \\
\hline $\operatorname{Sr} 31$ & Sr31/6*LMPG & $2-$ & $2-$ & 4 \\
\hline $\operatorname{Sr} 38$ & Trident & $; 1$ & 4 & 4 \\
\hline$M c N$ & McNair 701 & 4 & 4 & 4 \\
\hline
\end{tabular}

${ }^{a}$ ITs were accessed on seedlings at 14 days post inoculation using a 0 -to- 4 scale according to Stakman et al. (19), where ITs of $0, ;, 1,2$, or combinations are considered to be low ITs and ITs of 3 or higher are considered to be high. "N" or "C" denotes excessive necrosis or chlorosis, respectively. 
resistance gene or genes that are effective against race TRTTF. Race TRTTF has a virulence profile similar to race RRTTF (avirulent on $\mathrm{Sr} 9 e$ ), identified from stem rust collections in Ethiopia and Yemen in 2007 (4) and Pakistan in 2009 (6). Interestingly, both races have a uredinial morphology that is distinct from other common stem rust isolates in that the epidermal tissue over the uredinia has a delayed breakage and the color of uredinia is darker than normal (Fig. 1).

In addition to virulence to $\mathrm{Sr} 13$ and $\mathrm{Sr} 9 e$, race TRTTF exhibited a high IT (IT 3) to stem rust resistance conferred by the 1AL.1RS translocation (Table 2). This is the first known race with virulence to the stem rust resistance gene carried by this rye translocation, which represents one of the few effective genes to Ug99 in winter wheat cultivars in the United States (7). Race TRTTF, in combination with TTKSK, differentiates the stem rust resistance gene on 1AL.1RS from other stem rust resistance genes present on 1RS; namely, $\operatorname{Sr} 31$ and $\operatorname{Sr} 50(3,12)$. Additional data are needed to determine the genetic relationship between 1A.1R resistance and both Sr31 and Sr50. In the United States, current bread wheat breeding lines and cultivars with resistance to race TTKSK possess resistance genes including $\operatorname{Sr} 24, \operatorname{Sr} 36, \operatorname{SrTmp}$, and resistance on the 1A.1R translocation (7). Though race TRTTF is avirulent to $\operatorname{Sr} 31$,

Table 2. Infection types (ITs) observed on additional resistant lines using races JRCQC and TRTTF of Puccinia graminis f. sp. tritici identified from the 2009 Debre Zeit (Ethiopia) field nursery ${ }^{\mathrm{a}}$

\begin{tabular}{|c|c|c|c|}
\hline Gene & Line & JRCQC (09ETH08-3) & TRTTF (09ETH06-1) \\
\hline Resistant check & Iumillo & ;N & $11+; \mathrm{N}$ \\
\hline Susceptible check & Rusty & $3+$ & 4 \\
\hline $\operatorname{Sr} 13$ & Khapstein/9*LMPG & 3 & $3+$ \\
\hline Sr9e & Vernal & $3+$ & 3 \\
\hline Sr9e & K253/3*Steinwedel/8*LMPG & 4 & $2+$ \\
\hline $\operatorname{Sr} 13+\operatorname{Sr} 9 e$ & Leeds & 4 & ; \\
\hline $\operatorname{Sr} 22$ & SwSr22T.B. & $2-$ & $2-$ \\
\hline $\operatorname{Sr} 25$ & LcSr25Ars & $2+$ & $2+3-$ \\
\hline $\operatorname{Sr} 26$ & Eagle & 2 & $2-$ \\
\hline Sr27 & WRT 238-5 & ; & $2-$ \\
\hline $\operatorname{Sr} 32$ & ER 5155 & 2 & $2+$ \\
\hline Sr33 & Tetra Canthatch/Ae. Squarrosa & $; 2=$ & 2 \\
\hline $\operatorname{Sr} 35$ & $\mathrm{Mq}(2) 5 * \mathrm{G} 2919$ & 0 & 0 \\
\hline Sr37 & W3563 & 31 & $13+$ \\
\hline $\operatorname{Sr} 39$ & RL6082 & 0 & $2-$ \\
\hline Sr40 & RL6088 & $2-$ & 2 \\
\hline $\operatorname{Sr} 42$ & Norin 40 & $33+$ & 4 \\
\hline $\mathrm{Sr} 44$ & TAF 2 & ;N1 & $3+; \mathrm{N} 1$ \\
\hline Sr46 & AUS 18913 & 1 & $2-;$ \\
\hline $\operatorname{Sr} 47$ & DAS15 & $; 2=$ & $2-$ \\
\hline SrSatu & Satu & 0 & 0 \\
\hline $1 A .1 R$ & TAM 107 & $2-$ & 3 \\
\hline $\operatorname{Sr} 50$ & Fed $* 3 / G a b o * 51 B L .1 R S-1-1$ & $2-;$ & $; 1-$ \\
\hline
\end{tabular}

${ }^{a}$ ITs observed on seedlings at 14 days post inoculation using a 0 -to- 4 scale according to Stakman et al. (19), where ITs of 0 , ;, 1, 2, or combinations are considered to be low ITs and ITs of 3 or higher are considered to be high. "N" or "C" denotes excessive necrosis or chlorosis, respectively.

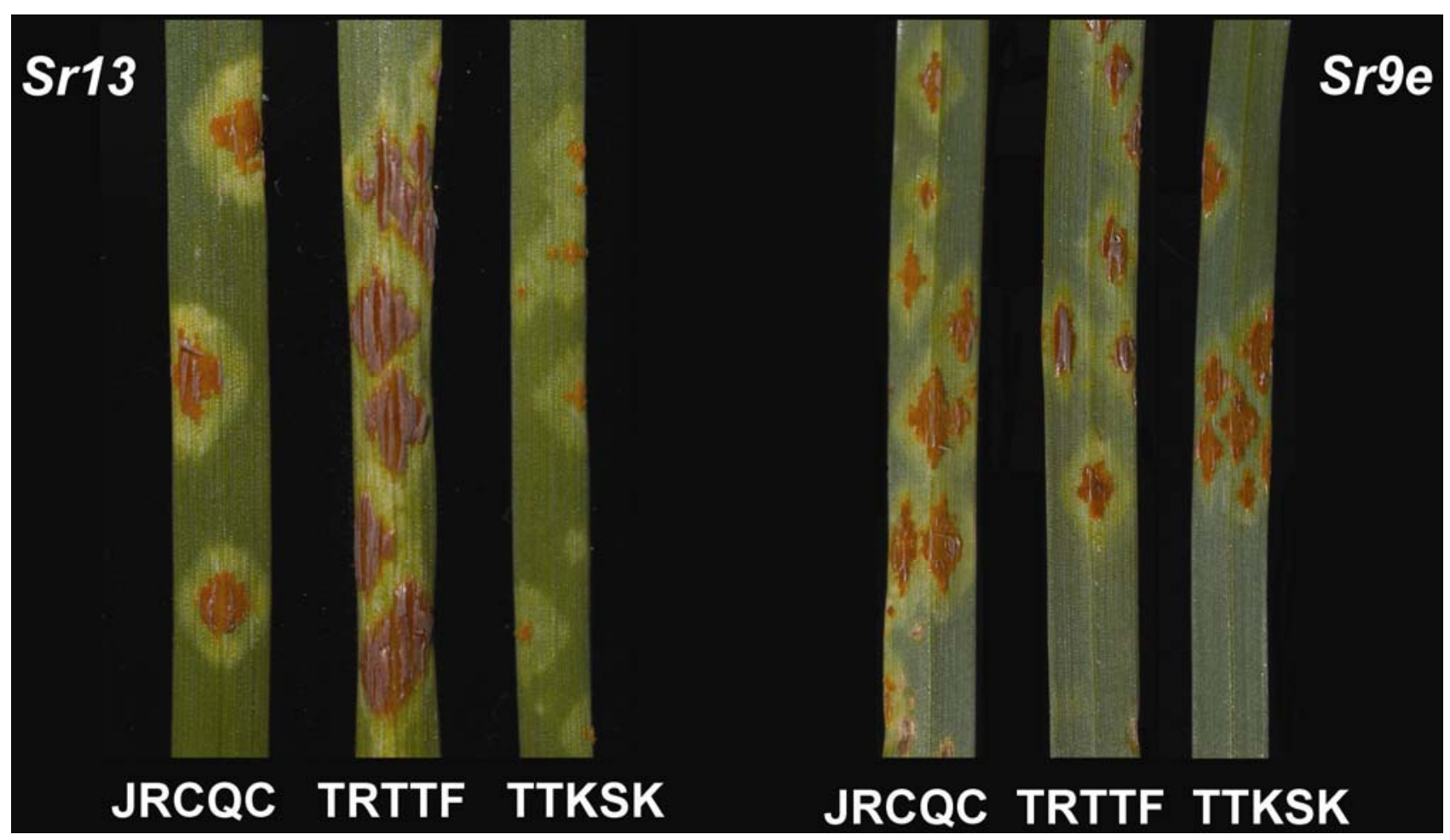

Fig. 1. Infection types produced by races JRCQC, TRTTF, and TTKSK of Puccinia graminis f. sp. tritici on Combination VII (Sr13) and Vernstein (Sr9e). 
Table 3. Disease reaction of durum wheat (Triticum turgidum subsp. durum) selected for resistant to moderately resistant to Puccinia graminis f. sp. tritici at the adult stage in field evaluations in Debre Zeit (Ethiopia) and at the seedling stage against races JRCQC, TRTTF, and TTKSK

\begin{tabular}{|c|c|c|c|c|c|c|}
\hline \multirow[b]{2}{*}{ Line ID } & \multirow[b]{2}{*}{ Type $^{c}$} & \multirow[b]{2}{*}{ Origin } & \multirow{2}{*}{$\begin{array}{c}\text { Adult }^{\mathrm{a}} \\
\text { Debre Zeit } 2009\end{array}$} & \multicolumn{3}{|c|}{ Seedlingb } \\
\hline & & & & JRCQC (09ETH08-3) & TRTTF (09ETH061) & TTKSK (04KEN156-4) \\
\hline CItr 6519 & Cultivar & North Dakota & $10 \mathrm{R}$ & 0 & 4 & ;CN / 3+ \\
\hline CItr 7287 & Cultivar & North Dakota & $20 \mathrm{MS}$ & 32 & 4 & 3 \\
\hline CItr 8123 & Landrace & Ethiopia & 40 MR-MS & $13+$ & ;2-N & $2-/ 2+2$ \\
\hline CItr 8214 & Cultivar & North Dakota & $10 \mathrm{MS}-\mathrm{MR}$ & $3+/ 3+; \mathrm{N}$ & $2+$ & $3+$ \\
\hline CItr 8634 & Landrace & Ethiopia & $20 \mathrm{MR}$ & $3+; 1$ & 4 & $3+$ \\
\hline CItr 11477 & Cultivar & North Dakota & $20 \mathrm{MS}$ & $; 13$ & 3 & 2 \\
\hline CItr 11541 & Cultivar & North Dakota & $20 \mathrm{MS}$ & 4 & 4 & $3+$ \\
\hline CItr 13245 & Cultivar & North Dakota & $10 \mathrm{R}-\mathrm{MR}$ & ; & $2-$ & $3+$ \\
\hline CItr 13247 & Cultivar & North Dakota & $15 \mathrm{MS}$ & $3+$ & $2+$ & $3+$ \\
\hline CItr 13333 & Cultivar & North Dakota & 5 R-MR & $2+$ & $; 1$ & $2-/ 3+$ \\
\hline CItr 13335 & Cultivar & North Dakota & $5 \mathrm{R}$ & 2 & 2 & $2-$ \\
\hline CItr 13338 & Breeding & Manitoba & $10 \mathrm{MR}-\mathrm{MS}$ & $0 ; / 2+$ & $2-;$ & ;CN / 2- \\
\hline CItr 13768 & Cultivar & North Dakota & 20 MS-MR & $3+$ & 0 & $2-\mathrm{N}$ \\
\hline CItr 14091 & Cultivated & Unknown & 5 MR-MS & 3 & $2+3-/ 22+$ & $\mathrm{X} / 3+$ \\
\hline CItr 14434 & Landrace & Ethiopia & 20 R-MR & $4 ; \mathrm{N} / ; \mathrm{N}$ & 4 & $33-$ \\
\hline CItr 14965 & Cultivated & Unknown & $10 \mathrm{MR}$ & ;N3+ & $3+$ & $3+$ \\
\hline CItr 15326 & Cultivar & North Dakota & $20 \mathrm{MR}$ & ;N & 0 & $2-$ \\
\hline CItr 15769 & Breeding & North Dakota & 15 MR-MS & ;N & ;2- & $2-2$ \\
\hline CItr 15814 & Breeding & North Dakota & T MR-MS & ;3 & ;N & $3+$ \\
\hline CItr 15892 & Cultivar & North Dakota & 15 MR-MS & ;N & ; & $2-$ \\
\hline CItr 17282 & Cultivar & North Dakota & $5 \mathrm{R}-\mathrm{MR}$ & $; 1+$ & ; & $3 / 2$ \\
\hline CItr 17283 & Cultivar & North Dakota & 15 MR-R & ;N / 3 & ;2- & $2-$ \\
\hline CItr 17284 & Cultivar & North Dakota & $5 \mathrm{MR}$ & ;1- & $; \mathrm{N}$ & $2-$ \\
\hline CItr 17337 & Cultivar & Saskatchewan & $15 \mathrm{MR}$ & 2 & $; 1 \mathrm{~N}$ & $2-$ \\
\hline CItr 17637 & Landrace & Ethiopia & $30 \mathrm{MS}$ & $33+/ 2$ & $22-$ & $22+/ 33+$ \\
\hline CItr 17748 & Cultivar & North Dakota & $10 \mathrm{MR}$ & ;1- & ; & $3+$ \\
\hline CItr 17789 & Cultivar & North Dakota & 10 R-MR & $; 1+\mathrm{N}$ & ;N & $2-$ \\
\hline PI 45441 & Cultivated & South Africa & 15 MR-MS & $32+$ & $2+3-$ & 2 \\
\hline PI 56251 & Landrace & Portugal & $20 \mathrm{MS}$ & $2+$ & $3-/ 2$ & $2 / 3+$ \\
\hline PI 61111 & Landrace & Georgia & $20 \mathrm{MS}$ & $3+3$ & $3+$ & $3+$ \\
\hline PI 61123 & Landrace & Kazakhstan & $10 \mathrm{MS}$ & $2+$ & 4 & $3+$ \\
\hline PI 61127 & Cultivar & Kyrgyzstan & $15 \mathrm{MS}$ & $3-$ & 4 & $3+$ \\
\hline PI 61176 & Landrace & Russian Fed. & $15 \mathrm{MS}$ & 4 & 3 & $3+$ \\
\hline PI 61351 & Cultivated & Hokkaido & $15 \mathrm{MS}$ & $3+1+$ & $3+$ & $3+$ \\
\hline PI 61873 & Cultivated & Morocco & $50 \mathrm{MS}-\mathrm{MR}$ & $2-$ & 2 & $2 / 3+$ \\
\hline PI 70718 & Landrace & Iraq & $20 \mathrm{MS}$ & $3+$ & 3-; & $3+$ \\
\hline PI 94694 & Landrace & Egypt & $15 \mathrm{MS}$ & $3-2+$ & $3+$ & $22-/ 2+3-$ \\
\hline PI 94726 & Landrace & Italy & $0 / 20 \mathrm{R}$ & $2-$ & 2 & $2-$ \\
\hline PI 94761 & Wild & Georgia & $\mathrm{T} \mathrm{R}$ & $33+$ & 4 & ;CN \\
\hline PI 113395 & Landrace & Egypt & $10 \mathrm{MR}$ & $2+; \mathrm{N} 3$ & $22+$ & $3-$ \\
\hline PI 113398 & Landrace & Egypt & 10 R-MR & 0 & $3+$ & 3 \\
\hline PI 166336 & Landrace & Turkey & 15 MR-MS & $3+/ 0$ & $3+$ & 3 \\
\hline PI 167270 & Landrace & Turkey & $5 \mathrm{MR}$ & $3+; \mathrm{N}$ & $3+$ & ; \\
\hline PI 168916 & Breeding & Mexico & $5 \mathrm{R}$ & 4 & $22+/ 3$ & 2 \\
\hline PI 168922 & Breeding & Mexico & $5 \mathrm{MS}-\mathrm{MR}$ & $2+/ ; \mathrm{N} / 0$ & 4 & 2 \\
\hline PI 178048 & Landrace & Turkey & $5 \mathrm{MS}$ & $2+$ & $3+$ & ; \\
\hline PI 178156 & Landrace & Turkey & $10 \mathrm{MS}$ & $3+1$ & $3+$ & ;CN \\
\hline PI 182668 & Cultivated & Lebanon & $5 \mathrm{MS}$ & $; 1+$ & $2+3-$ & $3-$ \\
\hline PI 184540 & Cultivated & Portugal & 10 MS-MR & $2+1$ & $3+/ 3 ; \mathrm{N}$ & $2-$ \\
\hline PI 184641 & Landrace & Portugal & $15 \mathrm{MS}$ & $2+$ & 4 & 4 \\
\hline PI 185300 & Breeding & Santa Fe & $25 \mathrm{MS}$ & $2++$ & 4 & $2-2$ \\
\hline PI 191183 & Landrace & Spain & $15 \mathrm{MS}$ & ;N3+ & $3+$ & $2-$ \\
\hline PI 191645 & Cultivated & Sao Paulo & $20 \mathrm{MR}-\mathrm{MS}$ & $2=$ & 2 & 2 \\
\hline PI 192051 & Landrace & Portugal & $5 \mathrm{R}$ & $2=$ & 4 & $2-; N$ \\
\hline PI 192399 & Landrace & Italy & $15 \mathrm{MS}$ & $1-;$ & $2-;$ & $2+3-$ \\
\hline PI 192711 & Cultivated & Gotland & $5 \mathrm{MS} / 40 \mathrm{~S}$ & $1 ; 3$ & $; 1$ & $; \mathrm{CN}$ \\
\hline PI 193920 & Landrace & Portugal & 30 MS-MR & $2-$ & $3+/ 2$ & $2-; / 3-$ \\
\hline PI 208910 & Landrace & Iraq & $30 \mathrm{MS}$ & $3+$ & 3 & 3 \\
\hline PI 210944 & Landrace & Cyprus & 15 MS-MR & $2-; / 33+$ & 4 & $3+$ \\
\hline PI 234386 & Landrace & Jordan & $30 \mathrm{MS}$ & $3-2 / 4$ & 4 & $3+$ \\
\hline PI 253801 & Landrace & Iraq & $30 \mathrm{MS}$ & ;13+ & $2+3-$ & $3+$ \\
\hline PI 264947 & Cultivated & Italy & $10 \mathrm{MS}$ & $2+2$ & $3-2$ & 4 \\
\hline PI 272476 & Breeding & Hungary & 30 MR-MS & $2-$ & 2 & $2-$ \\
\hline PI 272545 & Breeding & Hungary & $30 \mathrm{MS}$ & $3+1$ & 4 & $3+$ \\
\hline
\end{tabular}

${ }^{\text {a }}$ Plants evaluated for infection response (17) and severity following the modified Cobb scale $(13)$. $\mathrm{R}=$ resistant, $\mathrm{MR}=$ moderately resistant, MS = moderately susceptible, $\mathrm{S}=$ susceptible, and $\mathrm{T}=$ traces.

${ }^{\mathrm{b}}$ Infection types (ITs) observed on seedlings at 14 days post inoculation using a 0 -to-4 scale according to Stakman et al. (19), where ITs of 0 , ; 1 , 2, or combinations are considered to be low ITs and ITs of 3 or higher are considered to be high. "N" or "C" denotes excessive necrosis or chlorosis, respectively; / indicates that an accession was heterogeneous (the predominant type was given first); and - denotes missing data, frequently caused by poor viability of seed.

c Classification according to the United States Department of Agriculture-Agricultural Research Service, National Small Grain Collection (Aberdeen, ID). Cultivated $=$ uncertainty about the improvement status. 
Table 3. (continued from preceding page)

\begin{tabular}{|c|c|c|c|c|c|c|}
\hline \multirow[b]{2}{*}{ Line ID } & \multirow[b]{2}{*}{ Type $^{c}$} & \multirow[b]{2}{*}{ Origin } & \multirow{2}{*}{$\begin{array}{c}\text { Adult }^{\mathrm{a}} \\
\text { Debre Zeit } 2009\end{array}$} & \multicolumn{3}{|c|}{ Seedlingb } \\
\hline & & & & JRCQC (09ETH08-3) & TRTTF (09ETH061) & TTKSK (04KEN156-4) \\
\hline PI 272553 & Cultivated & Hungary & $40 \mathrm{MS}-\mathrm{MR}$ & ;N / 3 & $\mathrm{X}$ & $2-$ \\
\hline PI 274681 & Cultivated & Poland & $\mathrm{T} \mathrm{S}$ & ; & - & $3-c$ \\
\hline PI 278352 & Cultivated & Italy & T R & $1+13-$ & 2 & $2+3-$ \\
\hline PI 278380 & Cultivated & Malta & $15 \mathrm{MS}$ & ;N4 & $; 1$ & 3 \\
\hline PI278503 & Cultivated & Spain & $10 \mathrm{MS}$ & 31 & $1+$ & $2-$ \\
\hline PI 283854 & Cultivated & India & $10 \mathrm{MS}$ & 4 & 4 & $3+$ \\
\hline PI 286539 & Cultivar & Ecuador & $5 \mathrm{MR}$ & $; 1$ & 2 & $22+$ \\
\hline PI 295967 & Cultivar & Israel & $15 \mathrm{MS}-\mathrm{MR}$ & $3+$ & $23-;$ & 3 \\
\hline PI 298547 & Landrace & Ethiopia & $15 \mathrm{MR}$ & $; \mathrm{N}$ & $; 1$ & $2=$ \\
\hline PI 316092 & Breeding & Australia & $20 \mathrm{MS}$ & $2-$ & $2-$ & $2-$ \\
\hline PI 316096 & Cultivar & Australia & $15 \mathrm{MS}$ & 3 & $3+$ & 3 \\
\hline PI 320128 & Landrace & Ethiopia & $20 \mathrm{MR}-\mathrm{MS}$ & $; 2=$ & ; & $2-/ 2$ \\
\hline PI 324928 & Breeding & Argentina & $15 \mathrm{MR}$ & 1 & $2+3-$ & 3 \\
\hline PI 326315 & Cultivar & Azerbaijan & $\mathrm{T} \mathrm{S}$ & 31 & $3+$ & $3+$ \\
\hline PI 347217 & Landrace & Iran & $60 \mathrm{MS}-\mathrm{MR}$ & $2-$ & 4 & $2 / 3+$ \\
\hline PI 352317 & Breeding & Switzerland & $15 \mathrm{MR}$ & $; 1$ & $2-$ & $2-$ \\
\hline PI 352463 & Cultivar & Switzerland & $20 \mathrm{MR}$ & $3+$ & 2 & $33-$ \\
\hline PI 352512 & Wild & Switzerland & $5 \mathrm{R}$ & 3 & $2+$ & $2+$ \\
\hline PI 361149 & Landrace & India & $10 \mathrm{MR}$ & - & ;N1 & $2-$ \\
\hline PI 366110 & Landrace & Egypt & $20 \mathrm{MR}$ & $3+$ & 4 & $33+$ \\
\hline PI 367224 & Breeding & Italy & $10 \mathrm{R}-\mathrm{MR}$ & 1 & 2 & $3-2+$ \\
\hline PI 383416 & Breeding & France & 5 R-MR & 0 & 2 & $2++$ \\
\hline PI 384111 & Landrace & Ethiopia & $15 \mathrm{MR}$ & $3-2+$ & $2-$ & $2-$ \\
\hline PI 422412 & Cultivar & Australia & $30 \mathrm{MS}$ & $; 1$ & 2 & $2-$ \\
\hline PI 428539 & Cultivar & France & $\mathrm{T} \mathrm{R}$ & 0 & ; & $2-;$ \\
\hline PI 428541 & Cultivar & France & $10 \mathrm{MS}-\mathrm{MR}$ & 0 & $3-2$ & $3-3$ \\
\hline PI 428549 & Cultivar & France & $\mathrm{T} \mathrm{R}$ & $2-$ & $; 1$ & $2-$ \\
\hline PI 430747 & Landrace & Yemen & 40 MS-MR & $3-3$ & $22+$ & $2-$ \\
\hline PI 434919 & Landrace & Egypt & $30 \mathrm{MS}$ & $2-; / 3$ & $3+$ & 3 \\
\hline PI 434952 & Landrace & Egypt & $30 \mathrm{MS}-\mathrm{MR}$ & $3+$ & $3+$ & $3+$ \\
\hline PI 477881 & Landrace & Peru & $10 \mathrm{MR}$ & $33+$ & $3-2+$ & $3+3$ \\
\hline PI 478298 & Cultivar & North Dakota & 15 MR-MS & ;N & $; \mathrm{N}$ & $2-/ 3+$ \\
\hline PI 478304 & Breeding & North Dakota & 5 MR-MS & $3+/ 22+/ ; \mathrm{N}$ & ;N & $2-$ \\
\hline PI 478306 & Cultivar & Washington & $5 \mathrm{MS}$ & $2+$ & ;N & $2-$ \\
\hline PI 479916 & Landrace & Ethiopia & 20 MR-MS & $2-$ & $2-\mathrm{N}$ & $2-/ 3$ \\
\hline PI 479921 & Landrace & Ethiopia & $15 \mathrm{MR}$ & - & $2-\mathrm{N}$ & $2-$ \\
\hline PI 479923 & Landrace & Ethiopia & $20 \mathrm{MR}-\mathrm{R}$ & ;N & $2-; \mathrm{N}$ & $2-2$ \\
\hline PI 479956 & Landrace & Ethiopia & $15 \mathrm{MR}-\mathrm{R}$ & $; 2=/ 22-/ 3$ & ;N2- & $2-2$ \\
\hline PI 479959 & Landrace & Ethiopia & 40 MR-MS & $2=$ & ;N2- & $2-\mathrm{N}$ \\
\hline PI 480006 & Landrace & Ethiopia & 20 R-MR & ;N1 & ;N2- & $2-$ \\
\hline PI 480401 & Landrace & Ethiopia & $10 \mathrm{MS}-\mathrm{MR}$ & $; 1$ & $3-$ & $22+/ 2-$ \\
\hline PI 487290 & Landrace & Jordan & $15 \mathrm{MS}$ & $3+$ & 4 & $3+$ \\
\hline PI 497927 & Breeding & North Dakota & $5 \mathrm{MR}$ & 31 & 0 & $2-$ \\
\hline PI 506469 & Cultivar & Colorado & T R & $; 3+1$ & ; & $2-$ \\
\hline PI 506470 & Cultivar & Colorado & 5 MR-MS & $; 11+$ & 0 & $3+/ 2-$ \\
\hline PI 510694 & Breeding & North Dakota & $10 \mathrm{MR}-\mathrm{MS}$ & - & ;N & $2-$ \\
\hline PI 510696 & Cultivar & North Dakota & 5 MR-MS & ;N & ;N & $2-$ \\
\hline PI 519170 & Breeding & La Araucania & $10 \mathrm{MS}$ & 3 & $X-$ & ;3- \\
\hline PI 519171 & Breeding & La Araucania & $5 \mathrm{MR}$ & $; 1+$ & 2 & $3-2+$ \\
\hline PI 519445 & Breeding & North Dakota & $\mathrm{T} \mathrm{R}$ & $22+$ & ;N1 & $2-$ \\
\hline PI 519559 & Breeding & Syria & $5 \mathrm{MS}$ & ;N1+ & $2-;$ & $2-;$ \\
\hline PI 519619 & Breeding & Syria & $5 \mathrm{MS}$ & 4 & $22-$ & $2-$ \\
\hline PI 519639 & Breeding & Syria & $5 \mathrm{MS}$ & $3+$ & - & $2-; \mathrm{N}$ \\
\hline PI 519642 & Breeding & Kenya & $5 \mathrm{MS}$ & 4 & $22-$ & $2-$ \\
\hline PI 519811 & Cultivar & Italy & $10 \mathrm{MS}$ & $3+$ & $3+/ 2-$ & 3 \\
\hline PI 519832 & Cultivar & Lebanon & $15 \mathrm{MR}$ & ;N & $3-$ & $2-$ \\
\hline PI 520299 & Breeding & North Dakota & $5 \mathrm{MS}$ & $2-$ & ;N & $2-$ \\
\hline PI 520300 & Cultivar & California & $5 \mathrm{MR}-\mathrm{MS}$ & $3+$ & ;N & $2-;$ \\
\hline PI 520392 & Breeding & Mexico & $15 \mathrm{MS}$ & 2 & $2-$ & $22+$ \\
\hline PI 520413 & Breeding & Syria & $10 \mathrm{MR}-\mathrm{R}$ & $3+2$ & $2+$ & 3 \\
\hline PI 520518 & Breeding & North Dakota & 5 R-MR & ;N1 & ; & - \\
\hline PI 525395 & Cultivated & Morocco & $15 \mathrm{MS}$ & $3+3$ & $3+$ & 3 \\
\hline PI 537310 & Cultivar & Saskatchewan & $10 \mathrm{MR}$ & $2+$ & $; 1$ & $2-$ \\
\hline PI 572862 & Landrace & Azerbaijan & $20 \mathrm{MR}-\mathrm{MS}$ & 32 & 2 & $3-2+$ \\
\hline PI 573005 & Cultivar & Arizona & $10 \mathrm{MR}-\mathrm{MS}$ & $2+2$ & 2 & $2-\mathrm{N}$ \\
\hline PI 576787 & Landrace & Algeria & $25 \mathrm{MS}$ & $3+$ & 4 & - \\
\hline PI 585010 & Landrace & Ethiopia & $50 \mathrm{MS}-\mathrm{MR}$ & $3+; \mathrm{N}$ & $2-$ & - \\
\hline PI 585020 & Cultivated & Saudi Arabia & $20 \mathrm{MS}$ & $3-2+$ & $22+$ & $22+$ \\
\hline PI 601250 & Cultivar & Arizona & $15 \mathrm{R}-\mathrm{MR}$ & $1 ; \mathrm{N}$ & 2 & $2-$ \\
\hline PI 614658 & Cultivar & United States & $30 \mathrm{MS}$ & $3+$ & ; & $2-\mathrm{N} ; / 2+$ \\
\hline PI 623997 & Landrace & Iran & $20 \mathrm{MS}$ & $3+$ & $3+$ & 3 \\
\hline PI 624854 & Landrace & Iran & 15 MS-MR & 4 & $X-$ & 3 \\
\hline PI 636501 & Breeding & North Dakota & $15 \mathrm{MS}-\mathrm{MR}$ & $1+$ & ; & $3-/ 2-$ \\
\hline
\end{tabular}


it is virulent to $\operatorname{Sr} 36, \operatorname{SrTmp}$ and the $1 \mathrm{~A} .1 \mathrm{R}$ translocation that are effective against race TTKSK (Tables 1 and 2). Additional studies are needed to determine the percentage of bread wheat cultivars and breeding lines resistant to race TRTTF.

Stem rust isolates with virulence to $\mathrm{Sr} 9 e$ and $\mathrm{Sr} 13$ were first reported in Ethiopia in 1988 and 1989, respectively (5). They appear to be widespread, because Admassu et al. (2) described races with virulence to these two genes in the major wheat-growing regions in Ethiopia. Races JRCQC and TRTTF, described in this report, possess combined virulence on $\operatorname{Sr} 13$ and $\operatorname{Sr} 9 e$. This virulence combination may explain why the TTKSK-resistant durum entries in Njoro (Kenya) were susceptible in Debre Zeit (Ethiopia). Lines with $\operatorname{Sr} 13$ conferred a moderately resistant to moderately susceptible response when tested against race TTKSK in the Njoro nursery (8). However, lines and cultivars carrying Sr9e (Vernal, K253/3*Steinwedel/8*LMPG, Vernstein) and Srl3 (Khapstein/9*LMPG) exhibited a susceptible response in the Debre Zeit field (data not shown). The virulence combination to $\mathrm{Sr} 9 e$ and Srl3 is of particular concern because these two genes constitute major components of stem rust resistance in North American durum cultivars (11). Identification of novel resistance genes effective against races JRCQC, TRTTF, and TTKS is required to broaden the pool of resistance genes in durum wheat. The description of race TRTTF with virulence to several TTKSK-effective resistance genes should remind breeders and pathologists of the danger of deploying race-specific genes alone. Resistance gene combinations are necessary to provide long-lasting resistance to wheat stem rust.

A set of durum germplasm (137 entries) selected for resistant to moderately susceptible responses from the 2009 field nursery in Debre Zeit was evaluated for reaction to races JRCQC, TRTTF, and TTKSK at the seedling stage (Table 3 ). The numbers of accessions exhibiting low ITs to race JRCQC, TRTTF, and TTKSK were $96(70 \%), 89(65 \%)$, and $84(61 \%)$, respectively. Many of these lines appear to have race-specific genes and only 47 (34\%) accessions were resistant to all three races used in this study. These resistant lines could serve as sources of stem rust resistance for wheat-breeding programs. The genetics of resistance to races JRCQC and TRTTF in selected lines are being investigated. Twenty-six lines (19\%) that were resistant to moderately resistant in the field were susceptible to the three races at the seedling stage, suggesting that adult plant resistance might be present in these accessions. Most of the accessions (66\%) susceptible at the seedling stage were landraces and other cultivated materials (with uncertainty about the improvement status) from the Middle East and Caucasus regions, and landraces from North and East Africa (Table 3).

Race-typing experiments of isolates collected from Kenyan fields in recent years yielded only races belonging to the TTKS lineage $(9,10,22)$. However, surveys conducted in Ethiopia in the last 20 years had more diversity of $P$. graminis f. sp. tritici races $(1,2,20)$. Continuous and thorough race surveys in Ethiopia are required to have a clear picture of the virulence dynamics in the population of $P$. graminis $\mathrm{f}$. sp. tritici in the country. Identifying and monitoring movement of races with virulence to effective and widely used resistance genes is critical for defining breeding strategies for stem rust resistance in durum and bread wheat. Our results highlight the relevance of evaluating durum wheat lines for stem rust resistance in Ethiopia due to the presence of combined virulence to genes $\operatorname{Sr} 9 e$ and $S r 13$ in the $P$. graminis f. sp. tritici population.

\section{Acknowledgments}

This research is funded by the United States Department of Agriculture-Agricultural Research Service and the Durable Rust Resistance of Wheat, Cornell.
We thank L. Wanschura, S. Gale, C. Kebede, and B. Hibdo for their technical assistance.

\section{Literature Cited}

1. Admassu, B., and Emebet, F. 2005. Physiological races and virulence diversity of Puccinia graminis f. sp. tritici on wheat in Ethiopia. Phytopathol. Mediterr. 44:313-318

2. Admassu, B., Lind, V., Friedt, W., and Ordon, F. 2009. Virulence analysis of Puccinia graminis f. sp. tritici populations in Ethiopia with special consideration of Ug99. Plant Pathol. 58:362-369.

3. Anugrahwati, D. R., Shepherd, K. W., Verlin, D. C., Zhang, P., Mirzaghaderi, G., Alker, E., Francki, M. G., and Dundas, I. S. 2008. Isolation of wheat-rye 1 RS recombinants that break the linkage between the stem rust resistance gene $\mathrm{SrR}$ and secalin. Genome 51:341-349.

4. Fetch Jr., T. 2009. Stem rust-a wheat killer of global proportions. Can. J. Plant Pathol. 31:149.

5. Hulluka, M., Woldeab, G., Adnew, Y., Desta, R., and Badebo, A. 1991. Wheat pathology research in Ethiopia. Pages 173-218 in: Wheat Research in Ethiopia: A Historical Perspective. H. Gebre-Mariam, D. G. Tanner, and M. Hulluka, eds. IAR/CIMMYT, Addis Ababa, Ethiopia.

6. Iqbal, M. J., Ahmad, I., Khanzada, K. A., Ahmad, N., Rattu, A. R., Fayyaz M., Ahmad, Y., Hakro, A. A., and Kazi, A. M. 2010. Local stem rust virulence in Pakistan and future breeding strategies. Pak. J. Bot. 43:1999-2009.

7. Jin, Y., and Singh, R. P. 2006. Resistance in U.S. wheat to recent eastern African isolates of Puccinia graminis $\mathrm{f}$. sp. tritici with virulence to resistance genes Sr31. Plant Dis. 90:476-480.

8. Jin, Y., Singh, R. P., Ward, R. W., Wanyera, R., Kinyua, M., Njau, P., Fetch, T., Pretorius, Z. A., and Yahyaoui, A. 2007. Characterization of seedling infection types and adult plant infection responses of monogenic $\mathrm{Sr}$ gene lines to race TTKS of Puccinia graminis f. sp. tritici. Plant Dis. 91:1096-1099.

9. Jin, Y., Szabo, L. J., Pretorius, Z. A., Singh, R. P., Ward, R., and Fetch, T. Jr. 2008. Detection of virulence to resistance gene $S r 24$ within race TTKS of Puccinia graminis f. sp. tritici. Plant Dis. 92:923-926.

10. Jin, Y., Szabo, L. J., Rouse, M., Fetch, T., Jr., Pretorius, Z. A., Wanyera, R., and $\mathrm{Njau}, \mathrm{P}$. 2009. Detection of virulence to resistance gene Sr36 within the TTKS race lineage of Puccinia graminis f. sp. tritici. Plant Dis. 93:367-370.

11. Klindworth, D. L., Miller, J. D., Jin, Y., and Xu, S. S. 2007. Chromosomal locations of genes for stem rust resistance in monogenic lines derived from tetraploid wheat accession ST464. Crop Sci. 47:1441-1450.

12. Mago, R., Spielmeyer, W., Lawrence, G. J., Lagudah, E. S., Ellis, J. G., and Pryor, A. 2002. Identification and mapping of molecular markers linked to rust resistance genes located on chromosome $1 \mathrm{RS}$ of rye using wheat-rye translocation lines. Theor. Appl. Genet. 104:1317-1324.

13. Peterson, R. F., Campbell, A. B., and Hannah, A. E. 1948. A diagrammatic scale for estimating rust intensity of leaves and stem of cereals. Can. J. Res. Sect. C 26:496-500.

14. Pozniak, C. J., Reimer, S., Fetch, T., Clarke, J. M., Clarke, F. R., Somers, D., Knox, R. E., and Singh, A. K. 2008. Association mapping of UG99 resistance in a diverse durum wheat population. Pages 485-487 in: Proc 11th Int. Wheat Genet. Symp. R. Appels, R. Eastwood, E. Lagudah, P. Langridge, M. Mackay, L. McIntye, and P. Sharp, eds. Sydney University Press, Sydney, Australia.

15. Roelfs, A. P., Long, D. L., and Roberts, J. J. 1993. Races of Puccinia graminis in the United States during 1992. Plant Dis. 77:1122-1125.

16. Roelfs, A. P., and Martens, J. W. 1988. An international system of nomenclature for Puccinia graminis f. sp. tritici. Phytopathology 78:526-533.

17. Roelfs, A. P. Singh, R. P., and Saari, E. E. 1992. Rust Diseases of Wheat, Concepts and Methods of Disease Management. CIMMYT, Mexico.

18. Singh, R. P., Hodson, D. P., Huerta-Espino, J., Jin, Y., Bhavani, S., Njau, P., Herrera-Foessel, S. A., P. Singh, P., Singh, S., and Govindan, V. 2011. The emergence of Ug99 races of the stem rust fungus is a threat to world wheat production. Annu. Rev. Phytopathol. 49:465-481.

19. Stakman, E. C., Steward, D. M., and Loegering, W. Q. 1962. Identification and physiologic races of Puccinia graminis var. tritici. U. S. Dep. Agric. ARS E-617.

20. van Ginkel, M. G., Getnet, G., and Tessema, T. 1989. Stripe, stem and leaf rust races in major wheat producing areas in Ethiopia. IAR Newsl. Agric. Res. 3:6-8.

21. Visser, B., Herselman, L., Park, R. F., Karaoglu, H., Bender, C. M., and Pretorius, Z. A. 2011. Characterization of two new Puccinia graminis f. sp. tritici races within the Ug99 lineage in South Africa. Euphytica 179:119-127.

22. Wanyera, R., Kinyua, M. G., Jin, Y., and Singh, R. P. 2006. The spread of stem rust caused by Puccinia graminis f. sp. tritici, with virulence on $\mathrm{Sr} 31$ in wheat in Eastern Africa. Plant Dis. 90:113.

23. Zadoks, J. C., Chang, T. T., and Konzak, C. F. 1974. A decimal code for the growth stage of cereals. Weed Res. 14:415-421. 